\title{
Optimizing Dirac fermions quasi-confinement by potential smoothness engineering
}

\author{
B. Brun ${ }^{1}$, N. Moreau ${ }^{1}$, S. Somanchi², V.-H. Nguyen ${ }^{1}$, A. Mreńca-Kolasińska ${ }^{3}$, \\ K. Watanabe ${ }^{4}$, T. Taniguchi ${ }^{4}$, J.-C. Charlier ${ }^{1}$, C. Stampfer ${ }^{2} \&$ B. Hackens ${ }^{1}$ \\ ${ }^{1} I M C N / N A P S \& M O D L$, Université catholique de Louvain \\ (UCLouvain), B-1348 Louvain-la-Neuve, Belgium \\ 2 JARA-FIT and 2nd Institute of Physics - RWTH Aachen, Germany \\ 3 AGH University of Science and Technology - Kraków, Poland and \\ 4 National Institute for Materials Science, Namiki, Tsukuba, Japan*
}

(Dated: November 28, 2019)

With the advent of high mobility encapsulated graphene devices, new electronic components ruled by Dirac fermions optics have been envisioned and realized. The main building blocks of electron-optics devices are gate-defined p-n junctions, which guide, transmit and refract graphene charge carriers, just like prisms and lenses in optics. The reflection and transmission are governed by the p-n junction smoothness, a parameter difficult to tune in conventional devices. Here we create p-n junctions in graphene, using the polarized tip of a scanning gate microscope, yielding Fabry-Pérot interference fringes in the device resistance. We control the p-n junctions smoothness using the tip-to-graphene distance, and show increased interference contrast using smoother potential barriers. Extensive tight-binding simulation reveal that smooth potential barriers induce a pronounced quasi-confinement of Dirac fermions below the tip, yielding enhanced interference contrast. On the opposite, sharp barriers are excellent Dirac fermions transmitters and lead to poorly contrasted interferences. Our work emphasizes the importance of junction smoothness for relativistic electron optics devices engineering.

Keywords: Graphene, Dirac fermions optics, Scanning gate microscopy

\footnotetext{
*boris.brun@uclouvain.be, benoit.hackens@uclouvain.be
} 
In semiconductor technology, the charge carriers density profile governs the devices' properties. The so-called space charge zone is of fundamental importance in diodes, transistors or solar cells, and its control at the microscopic scale is a prerequisite to reach the desired properties. In graphene, a semi-metal hosting massless Dirac fermions [1], the density profile of a p-n junction plays a really peculiar role. Provided that electronic transport is ballistic, the ratio between the junction width and the Fermi wavelength governs the transmission and refraction properties of charge carriers. In particular, the relativistic Dirac fermions experience Klein tunneling when impinging perpendicularly on a p-n interface [2], which ensures them a perfect unitary transmission independent of the potential barrier height [3]. Additionally, a diverging flow of Dirac fermions is refocused at a p-n interface, similarly to photons entering a negative refraction index medium $[4,5]$, an effect denoted as Veselago lensing [6].

These exotic properties of graphene Dirac fermions led a plethora of electron-optics proposals and realizations, such as electronic optical fibers [7-10], lenses[11-17] and their advanced design to create highly focused electron beams [18], and even the combination of different optical elements to create a scanning Dirac fermions microscope [19]. Aside guiding, the partial reflection encountered at p-n interfaces has been proposed in the early days of graphene to create Fabry-Pérot interferometers with graphene n-p-n junctions [20]. These interferences have since then been observed in monolayer [21-27] as well as multilayer graphene $[28,29]$. In view of potential applications, complex n-p-n junction geometries that fully take advantage of these Fabry-Pérot interferences have already proven useful to build otherwise inaccessible graphene devices, such as reflectors [30, 31] and even transistors [32].

A Fabry-Pérot interferometer consists in two mirrors facing each other, and the transmission probabilities of these mirrors govern the interference fringes contrast. In graphene, the mirrors are materialized by two p-n junctions, and their transmission properties could in principle be tuned by controlling the p-n junctions width. However, p-n junctions in graphene are most often created by means of metallic or graphite gates, whose distance to the graphene plane is by essence fixed, so that the p-n junction width is fixed by the sample geometry. Here we use the polarized tip of a Scanning Gate Microscope (SGM) to induce a n-p-n junction, and take advantage of the SGM flexibility to control and characterize the p-n junctions width, independently of the potential barriers height.

Scanning gate microscopy (SGM) consists in scanning an electrically polarized metallic 
tip, acting as a local gate above a device's surface, and mapping out tip-induced device's conductance changes [33]. Initially developed to investigate transport in III-V semiconductor heterostructures [34-37], SGM brought spatially-resolved insights into transport phenomena occurring in graphene devices, through experiments, simulations and their combination [3848]. Recently, we demonstrated the viability of SGM to study ballistic transport in clean encapsulated graphene devices, and reported optical-like behavior of Dirac fermions using the tip-induced potential as a Veselago lens[49].

In the present paper, we show that the transmission probabilities of the p-n junctions can be controlled by tuning the SGM tip-to-sample distance. Analyzing our experimental findings in the light of tight-binding simulations, we show that the interferences contrast results from the Dirac fermions confinement efficiency, which is governed by the smoothness of the p-n interfaces.

The studied sample is based on graphene encapsulated between two $20 \mathrm{~nm}$-thick hBN layers, in which a $250 \mathrm{~nm}$-wide constriction is defined by etching [50]. The hBN/graphene/hBN stack lies on top of a highly doped $\mathrm{Si}$ substrate covered by a $300 \mathrm{~nm} \mathrm{SiO}_{2}$ insulating layer. This device is thermally anchored to the mixing chamber of a dilution refrigerator, in front of a cryogenic scanning probe microscope [51]. The device conductance $G$, or resistance $R$, is measured in 4-contacts configuration, by driving a $1 \mathrm{nA}$ current at a frequency of $77,7 \mathrm{~Hz}$, and recording the voltage between two opposite contacts using standard lock-in technique (as sketched in Fig. 1a). All the data presented here are recorded at a temperature of $100 \mathrm{mK}$, but global features were found almost independent of temperature up to $1 \mathrm{~K}$, and even a temperature of $4 \mathrm{~K}$ did not noticeably change the observed behavior. Most of the data presented here were recorded during a single cooldown (except Fig.1e-f), but this sample showed qualitatively similar behavior for 7 cooldowns.

The biased SGM tip locally changes the carrier density $n$, leading to a Lorentzian evolution of $n$, centered at the tip position. When placing the tip at the center of the constriction, a n-p-n or p-n-p configuration can be reached, depending on the tip voltage $V_{t i p}$ and backgate voltage $V_{b g}$. This is illustrated in Figure 1c showing resistance as a function of $V_{b g}$ and $V_{t i p}$ for a tip-to graphene distance $d_{t i p}=70 \mathrm{~nm}$, The n-p-n region, located at the lower left part of Fig. 1c, is decorated with a complex pattern of interleaved fringes, resulting from different types of interference phenomena. In the investigated geometry, one can indeed anticipate that, beside the tip-induced n-p-n or p-n-p junction, other confinements play a 
(a)

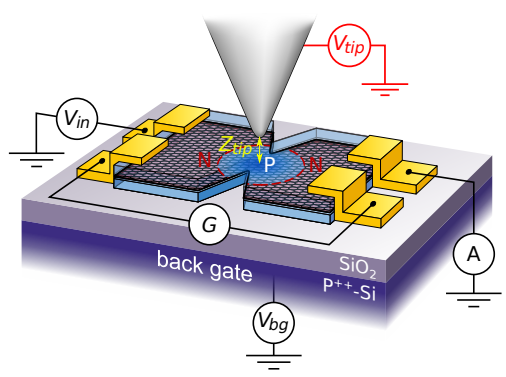

(b)

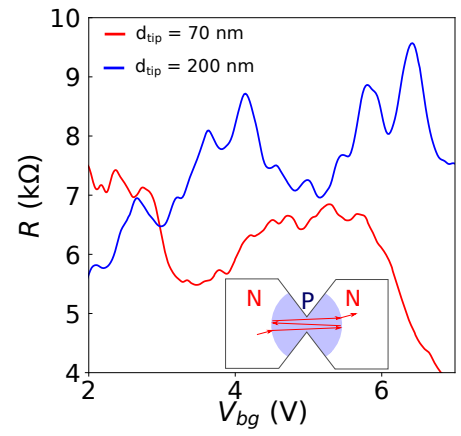

(c)

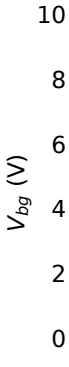

(d)

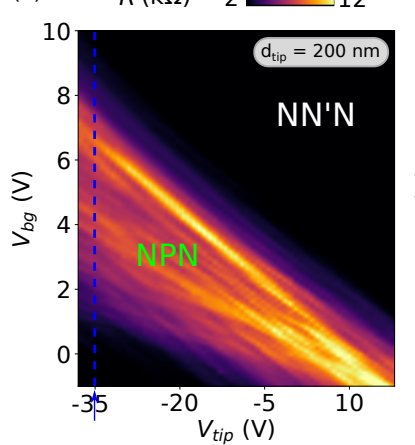

(e)
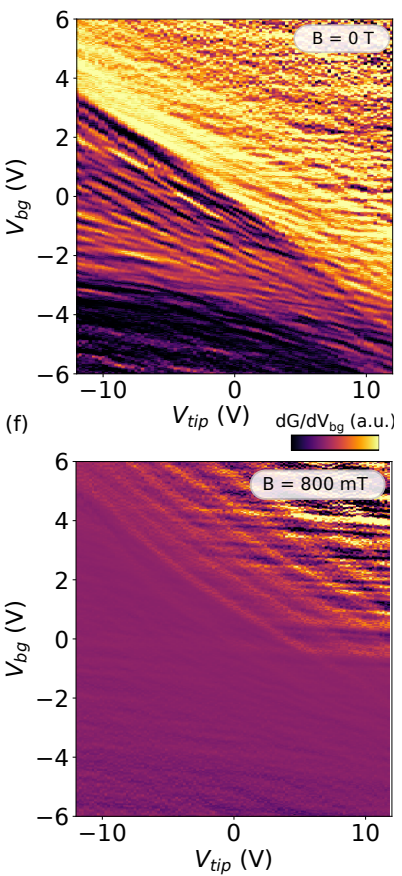

FIG. 1. Fabry-Pérot interference: (a) Scheme of the experiment: a biased AFM tip is placed above an encapsulated graphene constriction, creating a n-p-n or p-n-p junction. (b) Constriction resistance $R\left(V_{b g}\right)$ for a tip-to-graphene distance $d_{t i p}=70 \mathrm{~nm}\left(\right.$ red curve, $\left.V_{t i p}=-13 \mathrm{~V}\right)$; and $d_{t i p}=$ $200 \mathrm{~nm}$ (blue curve, $V_{t i p}=-35 \mathrm{~V}$ ). In both cases the tip is placed above the constriction center. (c) Resistance as a function of $V_{t i p}$ and $V_{b g}$, for $d_{t i p}=70 \mathrm{~nm}$, the tip being placed at the constriction center.(d) Resistance as a function of $V_{t i p}$ and $V_{b g}$, for $d_{t i p}=200 \mathrm{~nm}$. (e) Derivative of conductance versus $V_{t i p}$ and $V_{b g}$, recorded during a different cooldown and with a different tip, and $d_{t i p}=100$ nm. (f) Same configuration and cooldown as in (e), at a perpendicular magnetic field of $800 \mathrm{mT}$.

role and contribute to interferences in the map shown in Fig. 1c, such as the constriction defined by etching. Fortunately, increasing the tip-graphene distance to $d_{t i p}=200 \mathrm{~nm}$ yields a clearer picture, shown in Fig. 1d, with a much simpler fringe pattern (most of them essentially parallel to the n-p-n/n-n'-n limit). The visibility of the pattern is also enhanced by their stronger contrast, when compared to the pattern in Fig. 1c. In Figure 1b, we plot two profiles of resistance versus $V_{b g}$, for $d_{t i p}=70 \mathrm{~nm}$ (red curve) and $d_{t i p}=200 \mathrm{~nm}$ (blue curve) where $V_{\text {tip }}$ is adapted to reach comparable tip-induced density change (respectively $-13 \mathrm{~V}$ and $-35 \mathrm{~V})$. From this figure, the contrast of the oscillations appears clearly higher for a larger $d_{t i p}$, and a detailed discussion of the origin of this contrast enhancement is one 


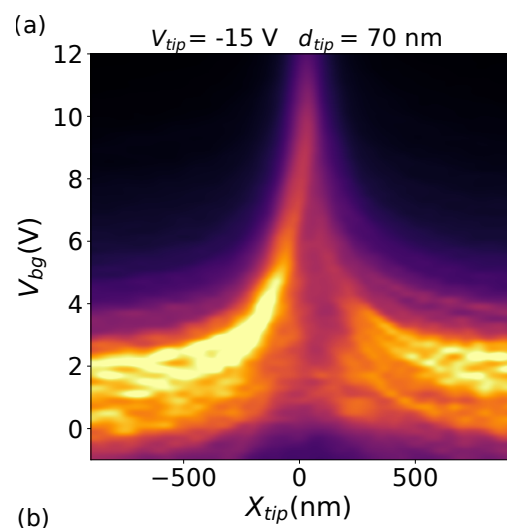

(b)

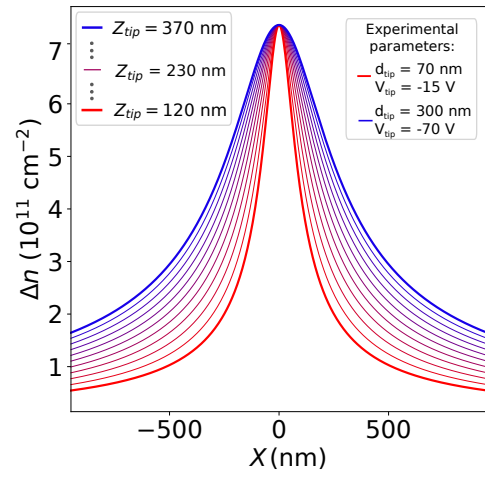

(c)
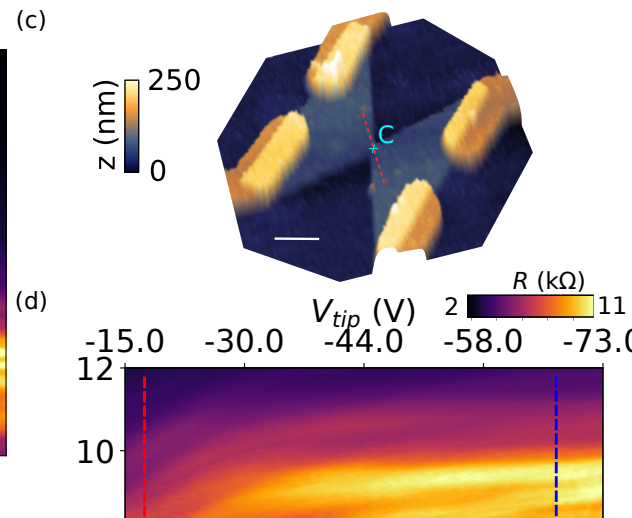

(e)

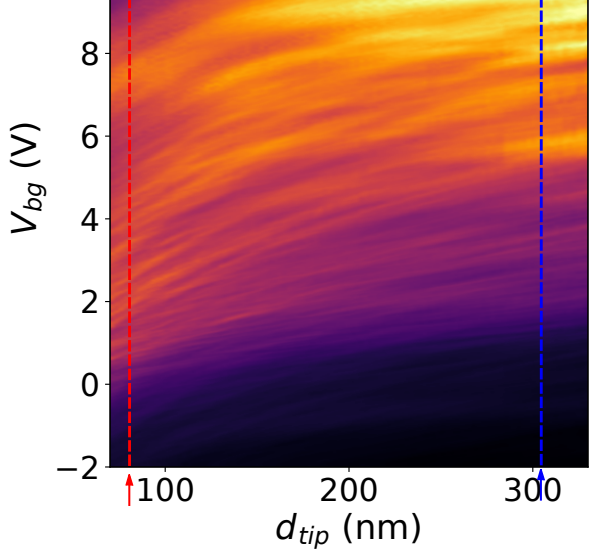

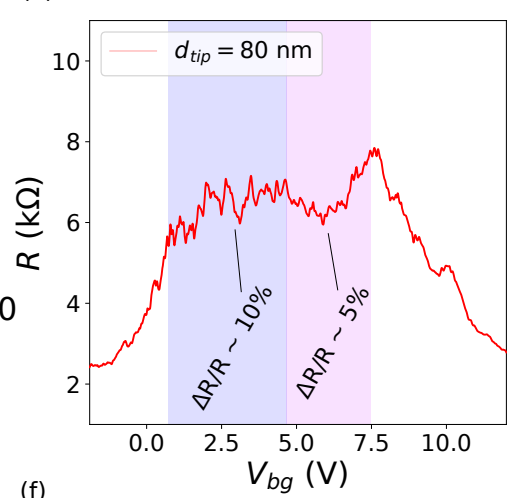

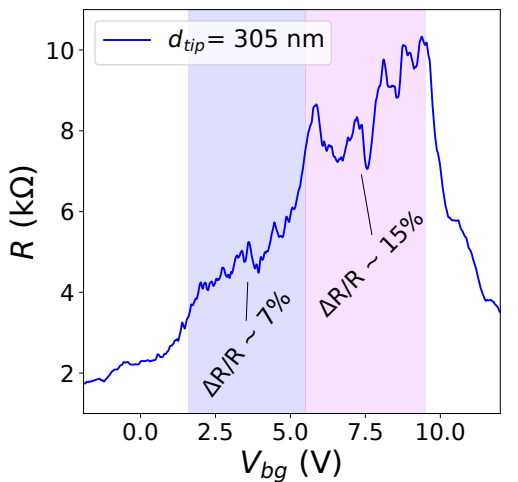

FIG. 2. Controlling the junction smoothness: (a) Resistance as a function of tip position along the blue dashed line in Fig. 2c $\left(x_{t i p}=0\right.$ being the center of the constriction), and $V_{b g}$. $d_{t i p}=70 \mathrm{~nm}, V_{t i p}=-15 \mathrm{~V}$. (b) Estimates of the tip-induced density change as a function of horizontal distance to the tip position $(x)$, for different couples of $d_{t i p}$ and $V_{t i p}$, chosen to keep the maximum density change $\Delta n^{\max }=7,5 \cdot 10^{11} \mathrm{~cm}^{-2}$ while changing the potential extension $R_{t i p}$. (c) AFM image of the device. Red dashed line represents the line scan used to evaluate the tip potential in (a) and (b). (d) Resistance as a function of $V_{b g}$ and $d_{t i p}$ (lower axis) and $V_{t i p}$ (upper axis). The tip is placed at the constriction center, both $d_{t i p}$ and $V_{t i p}$ are varied to keep a constant $\Delta n^{\max }=7,5.10^{11} \mathrm{~cm}^{-2}$ while varying $R_{t i p}$. (e,f): Resistance as a function of $V_{b g}$ for two different couples of $d_{t i p}$ and $V_{t i p}$, extracted from the colorplot in Fig. 2 d. (e) $d_{t i p}=70 \mathrm{~nm}$ and $V_{t i p}=-18$ V. (f) $d_{t i p}=305 \mathrm{~nm}$ and $V_{t i p}=-67 \mathrm{~V}$.

of the main focus of this paper.

It shall first be clarified that these oscillations correspond indeed to Fabry-Pérot interferences arising inside the tip-induced n-p-n region. Figures $1 \mathrm{e}$ and $1 \mathrm{f}$ (recorded during a different cooldown) illustrate the sensitivity of these interference fringes to a perpendicular magnetic field. Figure 1e presents the interference pattern recorded by placing the tip above 
the constriction center $\left(d_{t i p}=100 \mathrm{~nm}\right)$. The map in Fig. 1e displays the derivative of $G$ versus $V_{b g}$ to highlight the interference fringes, that appear similar to the ones observed in Fig. 1c-d. Fig. 1f shows that they have completely disappeared at a perpendicular magnetic field of $800 \mathrm{mT}$. From their characteristic decay field, one can infer that these fringes can be associated with a characteristic length, corresponding to a few hundreds nanometer-long cavity, compatible with the cavity formed in the tip induced n-p-n region, as sketched in the inset of Fig. 1b (see supplementary data for additional data and a more detailed discussion). In addition, an accurate determination of the tip-induced potential and an analytical calculation yielding the expected resonances positions in this potential profile agree well with the observed oscillations evolution, as detailed below. All these considerations provide strong evidence that the oscillations correspond to Fabry-Pérot oscillation in the tip-induced n-p-n region. In the remainder of this paper, we will discuss these interference fringes (Fig. 1c-e) and show that their visibility depends on the smoothness of the p-n junction, controlled by $d_{\text {tip }}$.

As a first step, one needs to precisely evaluate the tip-induced potential. This is done by scanning the tip along the blue dashed line Fig. 2c while varying $V_{b g}$, at fixed $V_{t i p}$ and tip-to-graphene distance $d_{t i p}$ (i.e. the same procedure described in ref. [49]). The resulting conductance map shown in Fig. 2a exhibits a resistance maximum that follows a Lorentzian shape, as the tip crosses the center of the constriction. This shape is directly related to the shape of the tip-induced potential, as it corresponds to the tip-induced change in the energy of the charge neutrality point at the location of the constriction, which governs the device resistance. Repeating this experiment for several values of $d_{t i p}$, and adapting $V_{\text {tip }}$ to keep a constant maximum density change below the tip $\Delta n^{m a x}$, we can fit the different density profiles under the tip influence, provided that the $V_{b g}$-axis is properly scaled to a density using the backgate lever-arm parameter (see supplementary data).

Considering the tip as a point charge, the expected tip-induced density change would write: $\Delta n(x)=\Delta n^{\max } /\left(1+x^{2} / Z_{\text {tip }}^{2}\right)$, where $x$ is the horizontal distance to the tip center, and $Z_{t i p}$ is the effective tip-to-graphene distance, i.e. $Z_{t i p}=d_{t i p}+a, a$ being the tip radius (a $=50 \mathrm{~nm}$ ). We define $R_{t i p}$ as the half-width at half-maximum (HWHM) of this density profile, that is in this expression given by the effective tip height $Z_{\text {tip }}$. Note that this textbook model underestimates the long-range tail of the tip-induced density change (see supplementary data). Accurately modeling the tip-induced potential yields a complex electrostatic problem 
[52-54], which is beyond the scope of the present paper. In turn, we model $\Delta n$ with the following phenomenological equation: $\Delta n(x)=\Delta n^{\max } /\left(\sqrt{1+3 x^{2} / Z_{\text {tip }}^{2}}\right)$, where we assume that the HWHM $R_{t i p}$ is given by $Z_{t i p}$ and is therefore known in the experiment, the only free parameter being $\Delta n^{\max }$. Fig. $2 \mathrm{~b}$ shows estimates of tip-induced density changes, for different couples of $V_{t i p}$ and $d_{t i p}$ leading to the same $\Delta n^{\max }$ (see supplementary data and movie for details).
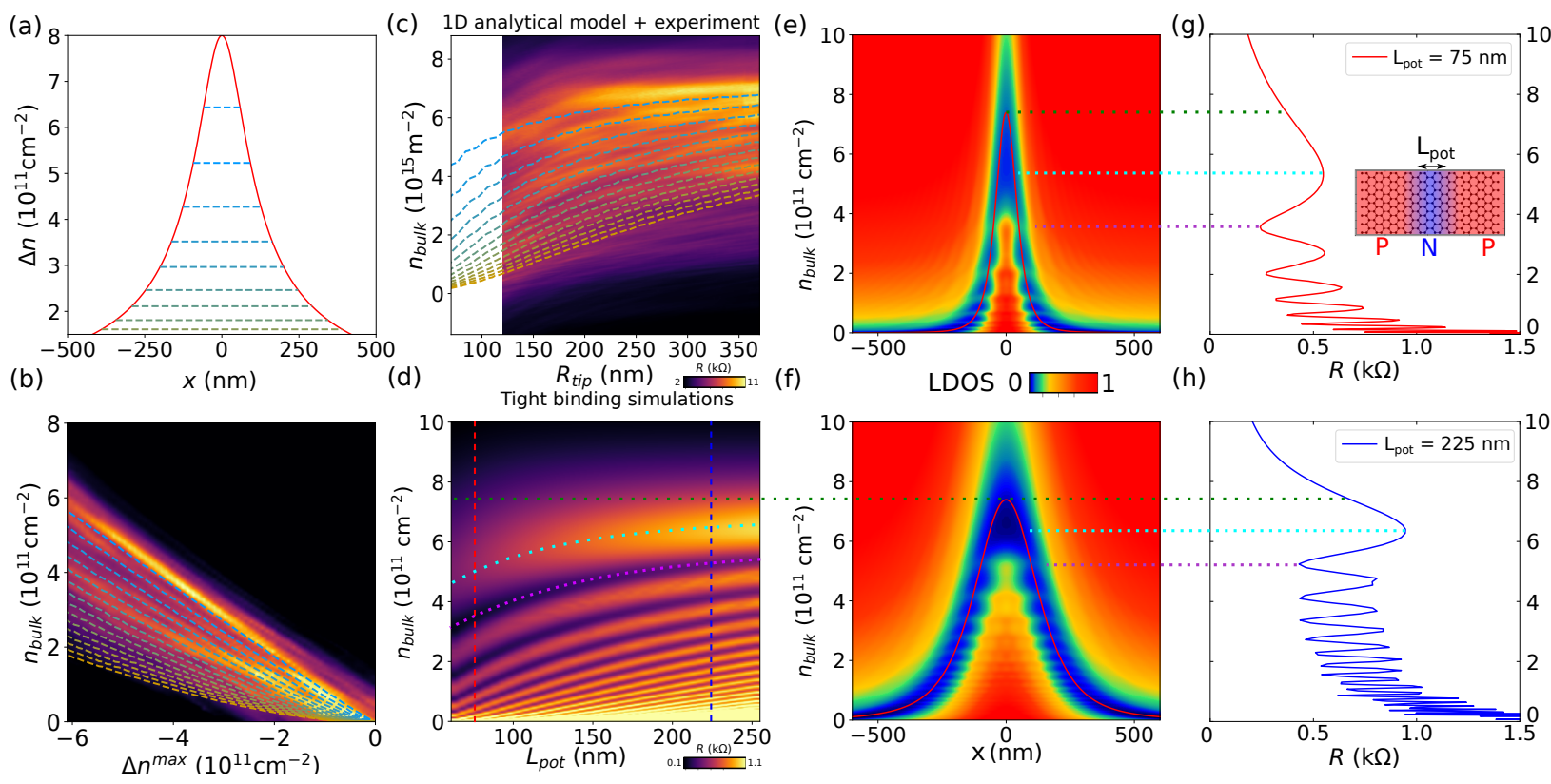

FIG. 3. Modeling the experiment: (a) Example of tip-induced density change, and corresponding resonant modes energies calculated from Eq.(1). (b) Position of the 10 first modes as a function of $\Delta n^{\max }$ and $n_{\text {bulk }}$, for $R_{t i p}=250 \mathrm{~nm}$, calculated from Eq.(1) and superimposed with the experimental data of Fig. 1d. (c) $1 \mathrm{D}$ analytical model of the position of Fabry-Prot modes calculated from Eq.(1), with $\Delta n^{\max }=7,5.10^{15} \mathrm{~m}^{-2}$. Dashed lines represent the bulk density corresponding to the 15 first resonances as a function of the potential extension $R_{t i p}$, superimposed with the experimental data of Fig. 2d. (d) Tight-binding model: simulated resistance as a function of the potential extension $L_{p o t}$ and bulk density $n_{b u l k}$, calculated for a graphene ribbon. (e) LDOS as a function of energy and $x$ position along the ribbon, showing the different resonances due to FP interferences, for a potential extension $L_{p o t}=70 \mathrm{~nm}$. Red line indicates the zero-density position. (f) Same calculation for $L_{p o t}=250 \mathrm{~nm}$. (g) Calculated resistance as a function of $n_{\text {bulk }}$ for the same potential as in (e). Inset: schematics of the tight-binding system. (h) Calculated resistance as a function of $n_{\text {bulk }}$ for the same potential as in (f). 
To study the influence of this potential extension on the Fabry-Pérot oscillations, we place the polarized tip on top of the constriction center (point $\mathrm{C}$ in Fig. 2c), and record the resistance as a function of $V_{b g}$, for different tip-to-graphene distance $d_{t i p}$. As $d_{t i p}$ is increased, we decrease $V_{\text {tip }}$ (towards more negative values) to keep a constant value of $\Delta n^{\text {max }}$, and vary only the smoothness of the p-n junctions through $R_{t i p}$. The resulting resistance map is plotted in Fig. 2d and constitutes the main result of this study, together with its detailed theoretical analysis. Figures $2 \mathrm{e}$ and $2 \mathrm{f}$ show the device resistance as a function of $V_{b g}$, for two extreme values of $R_{\text {tip }}$ in Fig. 2d. These two plots highlight two main features already visible in Fig. 2d, i.e.:

(i) The maximum value of the resistance increases with increasing $R_{t i p}$ as well as the density for which this maximum is reached.

(ii) The contrast of the Fabry-Pérot interference evolves in a different way for the lower energy modes observed at low $V_{b g}$ (they decrease in amplitude) and the higher energy ones, whose amplitude increases with $R_{t i p}$.

In order to understand these observations, we analyze the problem with two different approaches. The first one is analytic: we use the potential landscape evaluated from Fig. 2a and $2 \mathrm{~b}$, and follow the approach proposed in Ref.[55]. We consider the tip potential as varying only along $\mathrm{x}$-axis, and evaluate the position of the expected resonances from the simple equal phase condition:

$$
2 \int_{-L_{p}}^{L_{p}} k(x) d x=2 p \pi
$$

where $L_{p}$ is the position of zero charge density along x-axis which depends on the bulk density $n_{\text {bulk }}, p$ is a positive integer, and $k(x)$ is the position-dependent wave-vector evaluated from $n(x)$ provided that $k(x)=\sqrt{\pi n(x)}$. Fig. 3a shows a typical tip-induced density change and the position of the first resonant modes. In Fig. 3b, we calculate the expected position of the 10 first resonant modes for a tip potential extension of $250 \mathrm{~nm}$ as a function of $n_{\text {bulk }}$ and $\Delta n^{\max }$, and report them as dashed lines on top of the experimental data of Fig. 1d (where we have used the backgate and tip lever-arm parameters to convert the $V_{t i p}$ and $V_{b g}$ axis into carrier densities). There is a good qualitative correspondence between the evolution of the different modes and the experimental fringes, reinforcing the interpretation of their origin as Fabry-Pérot resonances inside the tip-induced n-p-n region. Using $n(x)$ measured for the different couples of $d_{t i p}$ and $V_{t i p}$, displayed in Fig. 2b, we also plot in Fig. 3c the evolution 
of the first 15 modes in the $\left(n_{b u l k}, R_{t i p}\right)$ plane, and find that they fall nicely on top of the experimental data of Fig. 2d, rescaling the vertical axis $V_{b g}$ to a density and the horizontal axes $d_{t i p}$ and $V_{t i p}$ to the tip potential extension $R_{t i p}$.

To go one step further in the understanding of the experimental fringes, we perform tightbinding simulations, using a home-made recursive Green functions code [56]. We study a simple graphene ribbon, to which we apply a potential of variable extension $L_{p o t}$ along transport direction, (see inset of Fig. 3g), with a smoothness governed by the exponent $\sigma$ :

$$
V(x)=\frac{V_{\max }}{1+\left(x / L_{p o t}\right)^{\sigma}}
$$

The ribbon width is fixed to $800 \mathrm{~nm}$ to avoid undesirable effects of transverse quantization (Fabry-Pérot resonances are insensitive to the ribbon width). We first consider a Lorentzian potential with $\sigma=2$, and calculate the ribbon resistance as a function of bulk density $n_{\text {bulk }}$ (i.e. the charge carrier density in the p region) and potential extension $L_{\text {pot }}$, while keeping a fixed value of $\Delta n^{\max }=7,5 \cdot 10^{11} \mathrm{~cm}^{-2}$. The result is plotted in Fig. 3d. First of all, it should be noted that the first resistance maximum (cyan dotted line on Fig. 3d) is not obtained for $n_{b u l k}=-\Delta n^{\max }$ (green dotted line). This is clarified in Fig. 3e, where we plot the local density of states (LDOS) in the graphene ribbon integrated over the transverse direction, as a function of $n_{\text {bluk }}$, aside with the resistance as a function of bulk density (Fig. 3g), for a potential extension $L_{p o t}=75 \mathrm{~nm}$. There is indeed a clear offset between the bulk density corresponding to the maximum of the tip-induced potential in Fig. 3e (indicated by a green dotted line) and the resistance maximum in Fig. 3g (indicated by a cyan dotted line). The resistance maximum is rather reached for a density $n_{\text {bulk }}$ yielding a minimum LDOS at the barrier center.

Figures $3 \mathrm{f}$ and $3 \mathrm{~h}$ present the same analysis for a larger potential extension. In this case, the bulk densities corresponding to $\Delta n^{\max }$ (in green) and to the minimum LDOS (in cyan) are closer to each other, but still do not match. The respective evolution of these two densities with the potential extension can be followed Fig. 3d, as the spacing between the green and cyan dotted lines, and is in good agreement with the evolution of the resistance maximum observed in the experiment, as visible in figures $2 \mathrm{~d}$ and $3 \mathrm{c}$.

A second interesting feature well captured by this toy model is the evolution of the first resonant mode energy, visible as the first resistance minimum indicated by purple dashed lines in Figs.3e-h, which follows roughly the same average evolution as the resistance 
maximum. This first Fabry-Pérot mode energy is reminiscent of the confinement energy due to the potential well created by the tip. In quantum mechanics, a famous textbook problem consists in finding the zero-point energy of a "particle-in-a-box", i.e. trapped in an infinite square potential of length $L$. The zero-point energy in the latter case emerges as a consequence of Heisenberg uncertainty principle, and increases with decreasing $L$ (as $L^{-2}$ for massive particles and $L^{-1}$ for massless Dirac fermions [57]). This distance of the first mode to the maximum of the tip potential is also clearly dependent on $R_{t i p}$ in the experiment, as visible ine Figs.2d and 3c. It provides a nice illustration of this textbook problem, poorly explored in the case of Dirac fermions due to the inherent difficulty to confine them.

Discrepancies are however visible between results from this ideal ribbon model and experimental data. First of all, additional resonances are present in the experiment. They could result from the transverse quantization inherent to the narrow constriction, intentionally suppressed in the tight-binding model by simulating a wide ribbon. These additional resonances could also arise from disorder, and the finite distance between the contacts and the constriction, that could lead to other Fabry-Pérot cavities. Secondly, the high resistance at low bulk density in the model is not present in the experiment. This can easily be understood as due to the experimentally measured finite resistance at the Dirac point, inherent to residual electron-hole puddles at low densities, whereas the tight-binding calculation in a homogeneous graphene ribbon predicts a much larger resistance of the bulk (and leads) close to the Dirac point. Both effects prevent the direct quantitative comparison of the interferences contrast in the experiment and the model presented in Fig. 3.

To better understand the influence of the tip potential extension, we perform additional tight-binding simulations and vary the potential steepness by changing the decay exponent $\sigma$ in equation (2). We first calculate the resistance of the ribbon as a function of bulk density and decay exponent, and plot the result in Fig. 4a. For three different decay exponents $(\sigma$ $=2,6,20)$ we extract the resistance as a function of $n_{b u l k}$ and plot the result in Fig. $4 \mathrm{~b}$. These two figures evidence that the potential smoothness is a key ingredient, that governs the Fabry-Pérot interference contrast. Indeed, the relativistic nature of graphene charge carriers makes sharp potential barriers highly transparent due to Klein tunneling. As a consequence, the Fabry-Pérot resonances in the LDOS are rather large and overlap (see Fig. 4c), owing to their hybridization with the Dirac continuum of the bulk. This weak confinement yields poorly contrasted Fabry-Pérot oscillations in the total resistance (red 

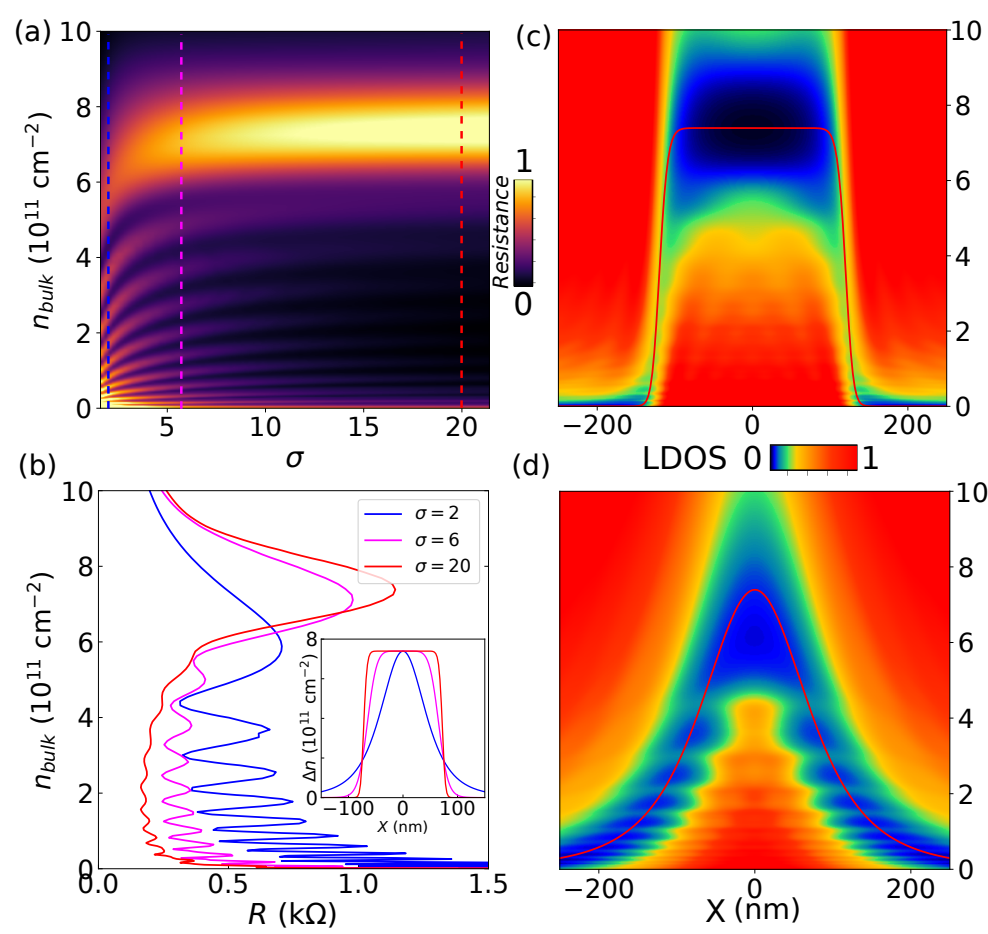

FIG. 4. Role of potential barriers smoothness: (a) Tight-binding simulation of the FabryPérot interference fringes evolution: resistance of a ribbon as a function of $n_{\text {bulk }}$ and potential smoothness $\sigma$, with fixed $\Delta n^{\max }=7,5 \cdot 10^{11} \mathrm{~cm}^{-2}$ and extension $L_{p o t}=100 \mathrm{~nm}$. (b) Profiles extracted from the dashed lines in Fig. 4a, corresponding to different decay exponent $\sigma$ from Lorentzian profile (blue, $\sigma=2$ ) to abrupt step (red, $\sigma=20$ ). (c) Calculated LDOS for $\sigma=20$ corresponding to the red resistance curve in Fig. 4b. (d) Calculated LDOS for $\sigma=2$, corresponding to the blue resistance curve in Fig. 4b.

curve Fig. 4b). In contrast, a smooth p-n junction (on the Fermi wavelength scale) is a poor Dirac fermions transmitter, so that two facing smooth p-n junctions can be used to confine Dirac fermions in a more efficient way. This can be seen Fig. 4d, where the LDOS in the case of a smooth n-p-n junction is plotted, and exhibits well defined resonant modes, giving rise to pronounced Fabry-Pérot oscillations in the resistance (blue curve Fig. 4b).

The confinement of Dirac fermions in p-n nano-islands and the resulting LDOS resonances have recently been explored in a set of beautiful scanning tunneling microscopy experiments [58-61]. Although our SGM experiment does not give direct access to the LDOS, it allows to probe transport through such islands and reveals the strength of LDOS resonances through Fabry-Pérot oscillations in the device resistance. Tight-binding simulations ex- 
plicitly confirm that the interference contrast is related to the LDOS resonances strength, themselves governed by the p-n junction smoothness, which can be easily tuned in SGM, as demonstrated here.

In conclusion, we defined a n-p-n junction in a high mobility graphene sample using the polarized tip of a scanning gate microscope. Oscillating patterns are observed in transport through the n-p-n junction that can be attributed to Fabry-Pérot interferences. By simultaneously varying the tip-to-graphene distance and tip voltage, one can control and characterize the $\mathrm{p}-\mathrm{n}$ junctions smoothness. In turn, this allowed to show that smoother $\mathrm{p}-\mathrm{n}$ junctions induce a larger contrast of the interference fringes. Using tight-binding simulations, we studied the influence of the p-n junctions smoothness on the LDOS resonances, resulting from the quasi-confinement of Dirac fermions within the tip-induced potential. These LDOS resonances amplitude can be explicitly linked to the visibility of the Fabry-Pérot oscillations. In the quest towards ever reduced graphene devices size, gates are often placed as close as possible to the graphene plane. The present study recalls that the gate dielectric thickness governs the p-n junction smoothness, which strongly influences the visibility of interferences. It then governs the efficiency of devices based on electron-optics concepts. This underlines that these distances have to be cleverly adjusted in the conception of relativistic electron optics devices.

\section{ACKNOWLEDGMENTS}

The present research was funded by the Fédération Wallonie-Bruxelles through the ARC Grant on 3D nanoarchitecturing of 2D crystals (No. 16/21-077) and from the European Union's Horizon 2020 Research and Innovation program (No. 696656). B.B. (research assistant), N.M. (FRIA fellowship), B.H. (research associate), V.-H.N. and J.-C.C. (PDR No. T.1077.15 and ERA-Net No. R.50.07.18.F) acknowledge financial support from the F.R.S.FNRS of Belgium. Support by the Helmholtz Nanoelectronic Facility (HNF), the EU ITN SPINOGRAPH and the DFG (SPP-1459) is gratefully acknowledged. Growth of hexagonal boron nitride crystals was supported by the Elemental Strategy Initiative conducted by the MEXT, Japan and JSPS KAKENHI Grant Numbers JP26248061, JP15K21722 and JP25106006. B.B aknowledges the use of Kwant [62] used to guide the experiment and 
cross-check tight-binding simulations.

\section{REFERENCES}

[1] K. S. Novoselov, A. K. Geim, S. V. Morozov, D. Jiang, M. I. Katsnelson, I. V. Grigorieva, S. V. Dubonos, and A. A. Firsov, Nature 438, 197 (2005).

[2] O. Klein, Zeitschrift für Physik 53, 157 (1929).

[3] P. E. Allain and J. N. Fuchs, The European Physical Journal B 83, 301 (2011).

[4] V. V. Cheianov, V. Fal'ko, and B. L. Altshuler, Science 315, 1252 (2007).

[5] S. P. Milovanovi, D. Moldovan, and F. M. Peeters, Journal of Applied Physics 118, 154308 (2015).

[6] V. G. Veselago, Soviet Physics Uspekhi 10, 509 (1968).

[7] C. W. J. Beenakker, R. A. Sepkhanov, A. R. Akhmerov, and J. Tworzydło, Phys. Rev. Lett. 102, $146804(2009)$.

[8] R. R. Hartmann, N. J. Robinson, and M. E. Portnoi, Phys. Rev. B 81, 245431 (2010).

[9] J. R. Williams, T. Low, M. S. Lundstrom, and C. M. Marcus, Nature Nanotechnology 6, 222 (2011).

[10] P. Rickhaus, M.-H. Liu, P. Makk, R. Maurand, S. Hess, S. Zihlmann, M. Weiss, K. Richter, and C. Schönenberger, Nano Letters 15, 5819 (2015).

[11] J. Cserti, A. Pályi, and C. Péterfalvi, Phys. Rev. Lett. 99, 246801 (2007).

[12] W. Mu, G. Zhang, Y. Tang, W. Wang, and Z. Ou-Yang, Journal of Physics: Condensed Matter 23, 495302 (2011).

[13] N. A. Garg, S. Ghosh, and M. Sharma, Journal of Physics: Condensed Matter 26, 155301 (2014).

[14] J.-S. Wu and M. M. Fogler, Phys. Rev. B 90, 235402 (2014).

[15] R. Logemann, K. J. A. Reijnders, T. Tudorovskiy, M. I. Katsnelson, and S. Yuan, Phys. Rev. B 91, 045420 (2015).

[16] M. Lu and X.-X. Zhang, Journal of Physics: Condensed Matter 30, 215303 (2018).

[17] S.-H. Zhang, W. Yang, and F. M. Peeters, Phys. Rev. B 97, 205437 (2018). 
[18] M.-H. Liu, C. Gorini, and K. Richter, Phys. Rev. Lett. 118, 066801 (2017).

[19] P. Bøggild, J. M. Caridad, C. Stampfer, G. Calogero, N. R. Papior, and M. Brandbyge, Nature Communications 8, 15783 (2017), article.

[20] A. V. Shytov, M. S. Rudner, and L. S. Levitov, Phys. Rev. Lett. 101, 156804 (2008).

[21] A. F. Young and P. Kim, Nature Physics 5, 222 (2009).

[22] J. Velasco, G. Liu, W. Bao, and C. N. Lau, New Journal of Physics 11, 095008 (2009).

[23] S.-G. Nam, D.-K. Ki, J. W. Park, Y. Kim, J. S. Kim, and H.-J. Lee, Nanotechnology 22, $415203(2011)$.

[24] P. Rickhaus, R. Maurand, M.-H. Liu, M. Weiss, K. Richter, and C. Schönenberger, Nature Communications 4, 2342 (2013), article.

[25] M. Oksanen, A. Uppstu, A. Laitinen, D. J. Cox, M. F. Craciun, S. Russo, A. Harju, and P. Hakonen, Phys. Rev. B 89, 121414 (2014).

[26] C. Handschin, P. Makk, P. Rickhaus, M.-H. Liu, K. Watanabe, T. Taniguchi, K. Richter, and C. Schönenberger, Nano Letters 17, 328 (2017).

[27] L. Veyrat, A. Jordan, K. Zimmermann, F. Gay, K. Watanabe, T. Taniguchi, H. Sellier, and B. Sacépé, Nano Letters 19, 635 (2019).

[28] A. Varlet, M.-H. Liu, V. Krueckl, D. Bischoff, P. Simonet, K. Watanabe, T. Taniguchi, K. Richter, K. Ensslin, and T. Ihn, Phys. Rev. Lett. 113, 116601 (2014).

[29] L. C. Campos, A. F. Young, K. Surakitbovorn, K. Watanabe, T. Taniguchi, and P. JarilloHerrero, Nature Communications 3, 1239 EP (2012), article.

[30] S. Morikawa, Q. Wilmart, S. Masubuchi, K. Watanabe, T. Taniguchi, B. Plaçais, and T. Machida, Semiconductor Science and Technology 32, 045010 (2017).

[31] H. Graef, Q. Wilmart, M. Rosticher, D. Mele, L. Banszerus, C. Stampfer, T. Taniguchi, K. Watanabe, J.-M. Berroir, E. Bocquillon, G. Fève, E. H. T. Teo, and B. Plaçais, Nature Communications 10, 2428 (2019).

[32] K. Wang, M. M. Elahi, L. Wang, K. M. M. Habib, T. Taniguchi, K. Watanabe, J. Hone, A. W. Ghosh, G.-H. Lee, and P. Kim, Proceedings of the National Academy of Sciences 116, 6575 (2019), https://www.pnas.org/content/116/14/6575.full.pdf.

[33] M. A. Eriksson, R. G. Beck, M. Topinka, J. A. Katine, R. M. Westervelt, K. L. Campman, and A. C. Gossard, Applied Physics Letters 69, 671 (1996). 
[34] M. A. Topinka, B. J. LeRoy, R. M. Westervelt, S. E. J. Shaw, R. Fleischmann, E. J. Heller, K. D. Maranowski, and A. C. Gossard, Nature 410, 183 (2001).

[35] M. P. Jura, M. A. Topinka, M. Grobis, L. N. Pfeiffer, K. W. West, and D. Goldhaber-Gordon, Phys. Rev. B 80, 041303 (2009).

[36] A. A. Kozikov, C. Rössler, T. Ihn, K. Ensslin, C. Reichl, and W. Wegscheider, New Journal of Physics 15, 013056 (2013).

[37] B. Brun, F. Martins, S. Faniel, B. Hackens, G. Bachelier, A. Cavanna, C. Ulysse, A. Ouerghi, U. Gennser, D. Mailly, S. Huant, V. Bayot, M. Sanquer, and H. Sellier, Nat. Commun. 5, 4290 (2014).

[38] S. Schnez, J. Güttinger, M. Huefner, C. Stampfer, K. Ensslin, and T. Ihn, Phys. Rev. B 82, 165445 (2010).

[39] N. Pascher, D. Bischoff, T. Ihn, and K. Ensslin, Applied Physics Letters 101, 063101 (2012), http://dx.doi.org/10.1063/1.4742862.

[40] A. G. F. Garcia, M. König, D. Goldhaber-Gordon, and K. Todd, Phys. Rev. B 87, 085446 (2013).

[41] D. Cabosart, A. Felten, N. Reckinger, A. Iordanescu, S. Toussaint, S. Faniel, and B. Hackens, Nano Letters 17, 1344 (2017).

[42] S. Bhandari, G.-H. Lee, A. Klales, K. Watanabe, T. Taniguchi, E. Heller, P. Kim, and R. M. Westervelt, Nano Letters 16, 1690 (2016).

[43] S. Xiang, A. Mreńca-Kolasińska, V. Miseikis, S. Guiducci, K. Kolasiński, C. Coletti, B. Szafran, F. Beltram, S. Roddaro, and S. Heun, Phys. Rev. B 94, 155446 (2016).

[44] K. Mreńca-Kolasińska, A.; Kolasiński and B. Szafran, Semiconductor Science and Technology 30, $085003(2015)$

[45] A. Mreńca-Kolasińska, S. Heun, and B. Szafran, Phys. Rev. B 93, 125411 (2016).

[46] A. Mreńca-Kolasińska and B. Szafran, Phys. Rev. B 96, 165310 (2017).

[47] M. D. Petrović, S. P. Milovanović, and F. M. Peeters, Nanotechnology 28, 185202 (2017).

[48] Z. Dou, S. Morikawa, A. Cresti, S.-W. Wang, C. G. Smith, C. Melios, O. Kazakova, K. Watanabe, T. Taniguchi, S. Masubuchi, T. Machida, and M. R. Connolly, Nano Letters 18, 2530 (2018).

[49] B. Brun, N. Moreau, S. Somanchi, V.-H. Nguyen, K. Watanabe, T. Taniguchi, J.-C. Charlier, C. Stampfer, and B. Hackens, Phys. Rev. B 100, 041401 (2019). 
[50] B. Terrés, L. A. Chizhova, F. Libisch, J. Peiro, D. Jörger, S. Engels, A. Girschik, K. Watanabe, T. Taniguchi, S. V. Rotkin, J. Burgdörfer, and C. Stampfer, Nature Communications 7, 11528 (2016), article.

[51] B. Hackens, F. Martins, S. Faniel, C. A. Dutu, H. Sellier, S. Huant, M. Pala, L. Desplanque, X. Wallart, and V. Bayot, Nat. Commun. 1, 39 (2010).

[52] L. M. Zhang and M. M. Fogler, Phys. Rev. Lett. 100, 116804 (2008).

[53] D. Żebrowski, A. Mreńca-Kolasińska, and B. Szafran, Phys. Rev. B 98, 155420 (2018).

[54] F. A. Chaves, D. Jimnez, J. E. Santos, P. Bøggild, and J. M. Caridad, Nanoscale 11, 10273 (2019).

[55] M. Drienovsky, F.-X. Schrettenbrunner, A. Sandner, D. Weiss, J. Eroms, M.-H. Liu, F. Tkatschenko, and K. Richter, Phys. Rev. B 89, 115421 (2014).

[56] V. H. Nguyen, A. Bournel, and P. Dollfus, Journal of Physics: Condensed Matter 22, 115304 (2010).

[57] S. Cho and M. Fuhrer, Nano Research 4, 385 (2011).

[58] C. Gutiérrez, L. Brown, C.-J. Kim, J. Park, and A. N. Pasupathy, Nature Physics 12, 1069 EP (2016), article.

[59] Y. Zhao, J. Wyrick, F. D. Natterer, J. F. Rodriguez-Nieva, C. Lewandowski, K. Watanabe, T. Taniguchi, L. S. Levitov, N. B. Zhitenev, and J. A. Stroscio, Science 348, 672 (2015).

[60] J. Lee, D. Wong, J. Velasco Jr, J. F. Rodriguez-Nieva, S. Kahn, H.-Z. Tsai, T. Taniguchi, K. Watanabe, A. Zettl, F. Wang, L. S. Levitov, and M. F. Crommie, Nature Physics 12, 1032 (2016).

[61] Y. Jiang, J. Mao, D. Moldovan, M. R. Masir, G. Li, K. Watanabe, T. Taniguchi, F. M. Peeters, and E. Y. Andrei, Nature Nanotechnology 12, 1045 (2017).

[62] C. W. Groth, M. Wimmer, A. R. Akhmerov, and X. Waintal, New Journal of Physics 16, 063065 (2014). 


\title{
Optimizing Dirac fermions quasi-confinement by potential smoothness engineering - Supporting information -
}

\author{
B. Brun ${ }^{1}$, N. Moreau ${ }^{1}$, S. Somanchi ${ }^{2}$, V.-H. Nguyen ${ }^{1}$, A. Mreńca-Kolasińska ${ }^{3}$, \\ K. Watanabe ${ }^{4}$, T. Taniguchi ${ }^{4}$, J.-C. Charlier ${ }^{1}$, C. Stampfer ${ }^{2} \&$ B. Hackens ${ }^{1}$ \\ ${ }^{1}$ IMCN/NAPS, Université catholique de Louvain (UCLouvain), B-1348 Louvain-la-Neuve, Belgium \\ 2 JARA-FIT and 2nd Institute of Physics - RWTH Aachen, Germany \\ ${ }^{3}$ AGH University of Science and Technology - Kraków, Poland \\ ${ }^{4}$ National Institute for Materials Science, Namiki, Tsukuba, Japan
}

November 21, 2019 


\section{Magnetic field dependence}
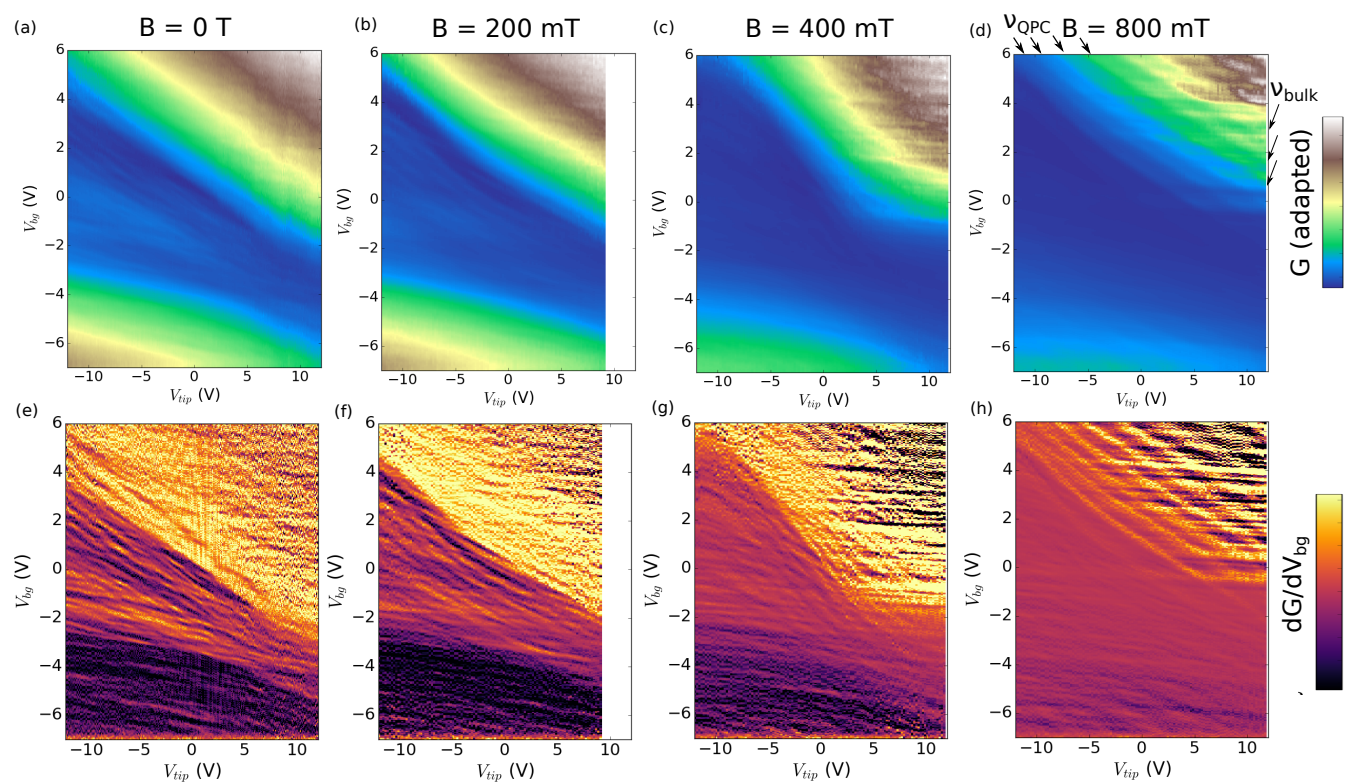

Figure S1: Evolution from Fabry-Pérot to quantum Hall regime: (a) Conductance $G$, as a function of $V_{t i p}$ and $V_{b g}$ for a tip-to-graphene distance of $90 \mathrm{~nm}$, recorded during a different cooldown, with respect to the data presented in the main text Fig.3, at zero magnetic field and a temperature of 4 K. (b) Same data set recorded at a perpendicular magnetic field $B$ of $200 \mathrm{mT}$. b) Same data set for $B=600 \mathrm{mT}$. (c) Same data set recorded for $B=800 \mathrm{mT}$. (d-f) Derivative of $G$ versus $V_{b g}$ for $B=0$, 200, 400 and $800 \mathrm{mT}$, corresponding to (a), (b), (c) and (d) respectively.

Figure S1 shows the evolution of the Fabry-Pérot interference pattern when appyling a perpendicular magnetic field, recorded during a different cooldown (compared to the data in the main text). The tip distance is set to $90 \mathrm{~nm}$, which in that experiment corresponded to a typical extension $R_{\text {tip }}=250 \mathrm{~nm}$ for a tip voltage of $-11 \mathrm{~V}$, as estimated following the procedure explained in Fig. 2a,b of the manuscript. Note that for a similar tip-to-graphene distance this potential extension was found to vary from a tip to another, which is expected from an electrostatic point of view since it depends on the tip apex radius, which can vary from one tip to another and for the same tip depending on its history. The Fabry-Pérot interference fringes recorded as a function of $V_{\text {tip }}$ and $V_{b g}$ therefore depend on the tip history, but was found consistent with the evaluated tip potential in most cooldowns.

At zero magnetic field (Fig.S1a and S1e), the Fabry-Pérot interferences are clearly visible in the $n-p-n$ configuration, and similar to the ones discussed in the manuscript. At $B=200 \mathrm{mT}$, the Fabry-Pérot interference fringes are still visible in the $n-p-n$ configuration (Fig.S1b and S1f). At a magnetic field of $400 \mathrm{mT}$ (Fig.S1c and S1g), the oscillations in the n-p-n configuration have almost shaded off, and new oscillations can be identified in the n-n'-n configuration. The oscillation 
parallel to the horizontal axis are insensitive to $V_{\text {tip }}$ and correspond to the bulk filling factor, which induces quantized conductance. The lines parallel to the n-n'-n correspond to the local Landau level below the tip, that differs from the bulk one, and also leads to a quantized conductance. For a typical density $n=5.10^{15} \mathrm{~m}^{-2}$, the cyclotron radius $r_{c}=\hbar * \sqrt{\pi n} /(e * B)$ equals $\sim 430 \mathrm{~nm}$ at $B=$ $200 \mathrm{mT}$, and $\sim 215 \mathrm{~nm}$ at $B=400 \mathrm{mT}$. The extension of the p-region below the tip (Rtip = $250 \mathrm{~nm}$ ) is therefore right in the range of the "cutoff" cyclotron radius, where Fabry-Pérot interferences vanish. This observation gives a strong indication that FP oscillations are forming below the tip. At a magnetic field of $800 \mathrm{mT}$ (Fig.S1d and S1h), the Fabry-Pérot interferences are not visible anymore, and the characteristic pattern of a n-n'-n constriction under magnetic field is clearly visible in the n-n'-n configuration and in the $p-p^{\prime}-p$ configuration, though shallower.

\section{Modeling the tip-induced potential: electrostatic considerations}

In an attempt to model the tip-induced potential decay with distance, we first consider the tip as a charged sphere of radius $a=50 \mathrm{~nm}$, and potential $V_{\text {tip }}$ (as shown in Fig. S2a). If the tip radius $a$ can be neglected compared to the tip-to graphene distance $d_{t i p}$, the charged sphere can be modeled as a point charge of charge $Q=4 \pi \epsilon_{0} V_{\text {tip }} a$. We neglect the contribution of dielectric layers (h-BN and $\mathrm{SiO}_{2}$ ) since they influence only the potential maximum value $V_{\text {max }}$, that will be treated as the only free parameter. Placing the point charge at abscissa $x=0$, and at a vertical distance $Z_{\text {tip }}=d_{t i p}+a$, the resulting potential at the graphene plane level can be expressed as:

$$
V(x)=\frac{V_{\max }}{\sqrt{x^{2}+Z_{t i p}^{2}}}
$$

Using $V_{\max }$ as the only free parameter, we fit the tip-induced density changes $\Delta n(x)$ measured experimentally as described in Fig. 1c of the main text, and reproduced for different tip distances and voltages in the colorplots of Fig. S2(b-d). (Note that the tip-induced density change $\Delta n(x)$ is linked to $V(x)$ as $\Delta n(x)=\left(\frac{V(x)}{\pi \hbar v_{F}}\right)^{2}$, due to the Dirac nature of charge carriers.) This simple model slightly underestimates the long tail of the tip-induced density change (for $|x|>0.5 \mu \mathrm{m}$ ).

To go one step further in the modeling, we take into account the backgate as an infinite plane at a distance $d_{b g}=320 \mathrm{~nm}$ below the graphene plane, as sketched Fig. S2e. We then have to take into account the contribution of an image charge $-Q$ at a distance $Z_{t i p}+2 Z_{b g}$ from the graphene plane. The total potential therefore writes:

$$
V(x)=V_{\text {max }} \times\left(\frac{1}{\sqrt{x^{2}+Z_{\text {tip }}^{2}}}-\frac{1}{\sqrt{x^{2}+\left(Z_{t i p}+2 Z_{b g}\right)^{2}}}\right)
$$

We adapt $V_{\max }$ to match the experimental data at $\mathrm{x}=0$ and plot the results Fig. S2(f-h). The tail of the potential appears even more underestimated than with the simple charge model proposed in Eq.(1). This is expected since in this more accurate modeling the effect of the tip potential is screened by the backgate. Considering screening by the graphene charge carriers themselves would lead to a much more complicated problem, consisting in solving iteratively the Poisson equation in the Thomas-Fermi approximation, in a realistic environment including all dielectric layers permittivities. This is far beyond the scope of this work, and is not expected to give more 


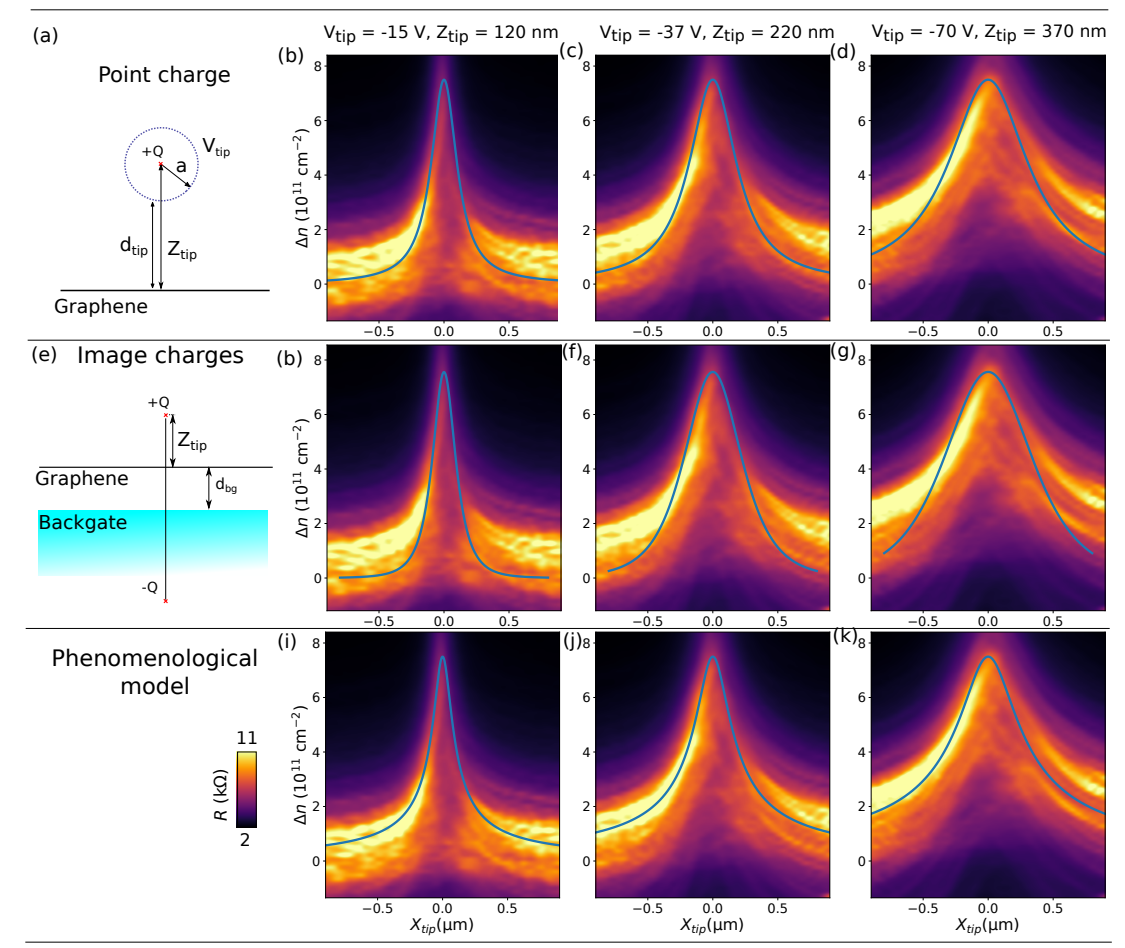

Figure S2: Different electrostatic models: (a) Scheme of a uniformly charged sphere, reduced to a punctual charge, at a distance $Z_{\text {tip }}$ from the graphene plane. (b) Same data as Fig.2a in the manuscript: resistance as a function of $X_{t i p}$, tip position along blue dashed line Fig.2c, and density, for $V_{t i p}=-15 \mathrm{~V}$ and $Z_{\text {tip }}=120 \mathrm{~nm}$. The result from the point charge model from Eq.(1) is superimposed as a blue curve. (c) Same data recorded for $V_{\text {tip }}=-37 \mathrm{~V}$ and $Z_{\text {tip }}=220 \mathrm{~nm}$, superimposed on the corresponding model from Eq.(1). (d) Same as (b-c) for $V_{t i p}=-70 \mathrm{~V}$ and $Z_{\text {tip }}=370 \mathrm{~nm}$. (e) Scheme of a point charge, at a distance $Z_{t i p}$ from the graphene plane, itself at a distance $Z_{b g}$ above the equipotential backgate plane. (f-h) Same data as in (b-d) superimposed on the results of the image charge model given by Eq.(2). (i-k) Same data as in (b-d) superimposed on the results of the phenomenological model used in the manuscript recalled in Eq.(3).

accurate results, since at low bulk densities the Fermi wavelength overcomes the potential extent, rendering the Thomas-Fermi approximation (small Fermi wavelength) very inappropriate.

However, intuition indicates that solving this considerably more complicated problem for a punctual charge would give an even worse result, since screening from the graphene charge carriers would lead to an even more rapidly decaying potential away from the tip center. The most questionable assumption in the above models resides in considering the tip as a point charge. In fact, in our experiment, the tip is a commercial HQ:CSC17/Pt tip from Mikromash, and we know that in the experimental conditions (i.e. after few weeks of experiment), the tip radius $a$ is typically $50 \mathrm{~nm}$. This cannot be neglected compared to $d_{\text {tip }}$ (as low as $70 \mathrm{~nm}$ for the closest distance). 
A more accurate tip modeling would therefore consist in considering a sphere or even a cone, and solve iteratively the complete problem with a 3D Poisson solver, as is often necessary to accurately model the tip-induced potential in SGM experiments [Iordanescu et al., in preparation].

In turn, we use here a third and more simple approach to evaluate the tip-induced potential through a phenomenological equation, and consider the tip-induced potential as leading to a density change:

$$
\Delta n(x)=\frac{\Delta n^{\max }}{\sqrt{1+3 \frac{x^{2}}{Z_{\text {tip }}^{2}}}}
$$

We adapt $\Delta n^{\max }$ to match experimental data at $\mathrm{x}=0$ and keep the known experimental $Z_{t i p}$, which in this equation gives the half width at half maximum of the profile of the tip-induced density change, as in Eq.(1). The results are plotted in Fig. S2(i-k), and match rather well the experimental measurements, given the fact that there is only one fitting parameter. This type of density evolution for Dirac fermions would correspond to a potential $V \propto\left(3 x^{2}+Z_{\text {tip }}^{2}\right)^{-\frac{1}{4}}$. Though this has no theoretical ground, the lower decay exponent accounts for the long tail of the tip-induced potential observed experimentally, that we attribute to the tip geometry. We therefore kept this phenomenological potential in the first part of the manuscript to evaluate the expected Fabry-Pérot resonances positions, since this model is the one that describes the most accurately the experimental tip potential (though the point charge model qualitatively works too).

\section{Determination of the lever-arm}

The determination of the lever-arm was done during another cooldown of the same sample, following the procedure described in this section. In order to convert the back gate voltage $V_{b g}$ into a charge carrier density $n_{2 D}$, we need to evaluate the lever arm defined by $C=n / \Delta V$ where $\Delta V=V_{b g}-V_{C N P}$ with $V_{C N P}=-1 \mathrm{~V}$ in our sample. This can be achieved by two different methods:

- From a simple capacitor model with $d_{h-B N}=30 \mathrm{~nm}$ and $d_{S i O_{2}}=300 \mathrm{~nm}$, the lever-arm is $\mathrm{C}=6.1-6.7 \times 10^{14} \mathrm{~m}^{-2} \mathrm{~V}^{-1}$, depending on h-BN dielectric constant $\left(\epsilon_{\mathrm{h}-\mathrm{BN}}=2-4\right)$.

- From Shubnikov-de Haas (SdH) oscillations, the Landau levels positions in the $\left(V_{g}, B\right)$ plane are given by the expression

$$
B_{v}=\frac{\pi \hbar}{2 e v} C \Delta V
$$

where $v$ is the filling factor. By fitting the Landau levels positions in the conductance map in the $\left(V_{g}, B\right)$ plane (Fig. S3b), measured in the bulk of the sample (see Fig. S3a for the measurement configuration), we obtain $C=7.4 \pm 0.2 \times 10^{14} \mathrm{~m}^{-2} \mathrm{~V}^{-1}$.

\section{Mobilities}

Charge carriers mobilities are extracted from linear fits to the $G$ vs $n$ curve in the vicinity of the charge neutrality point (i.e. the Dirac cone). Note that we measure $G$ in the two-contacts configuration depicted in Fig. S3a, taking into account a total series resistance of $2 \mathrm{k} \Omega$ corresponding 
(a)

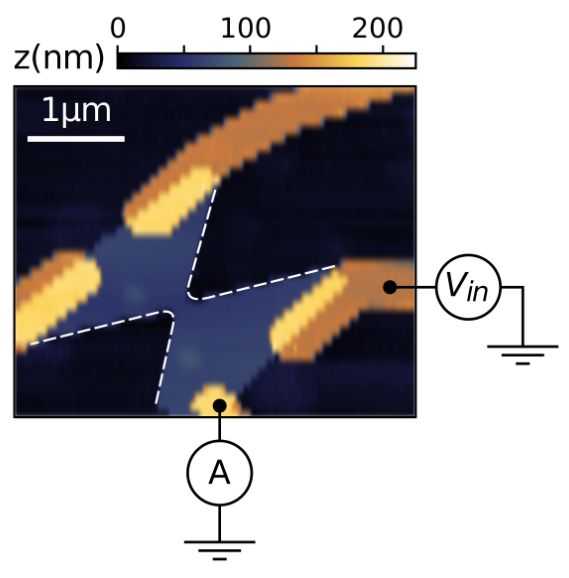

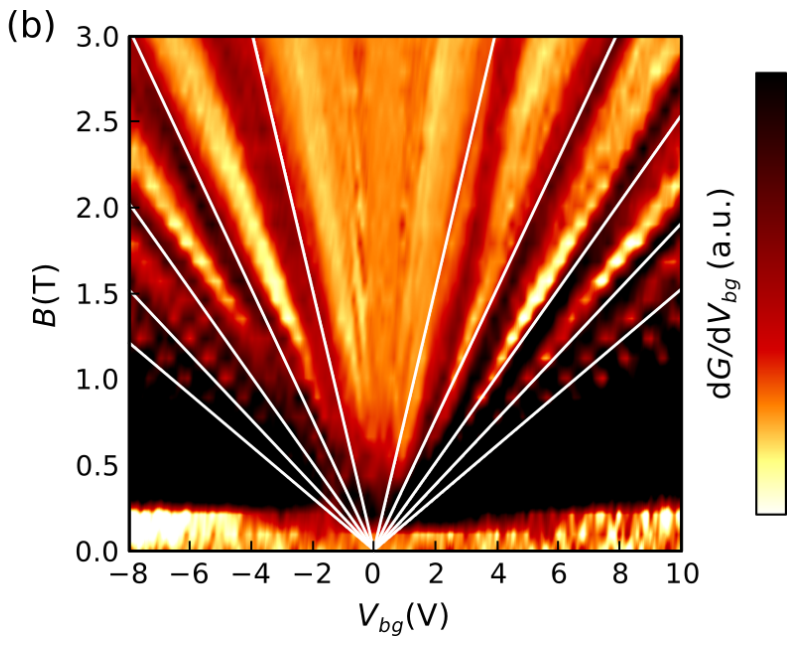

Figure S3: Lever-arm parameter of the back-gate. (a) AFM topography image of the graphene device. The two contacts used for the transconductance measurement shown in (b) are below the constriction, in yellow, and separated from each other by a distance of $800 \mathrm{~nm}$. (b) Transconductance $\partial G / \partial V_{b g}$ as a function of $V_{b g}$ and $B$, with a superimposed fit to the expression of Landau level position, depending on the lever arm (see text).

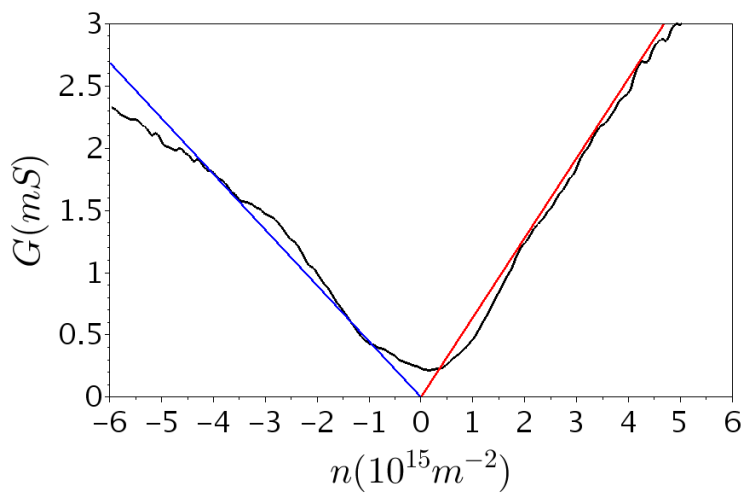

Figure S4: Electron and holes mobilities from the Dirac cone slope. Dirac cone measured in the same configuration as Fig. S3a. Two linear fits allow to calculate lower bounds for the electron and holes mobilities.

to the electrical filters on the measurement setup. This yields lower bounds for the mobilities : $\mu_{e} \sim 40000 \mathrm{~cm}^{2} \mathrm{~V}^{-1} \mathrm{~s}^{-1}$ for electrons and $\mu_{h} \sim 28000 \mathrm{~cm}^{2} \mathrm{~V}^{-1} \mathrm{~s}^{-1}$ for holes. 